\title{
PHOTOMETRIC OBSERVATIONS OF VISUAL DOUBLE STARS \\ BY THE AREA SCANNING TECHNIQUE
}

\author{
OTTO G. FRANZ \\ Lowell Observatory, Flagstaff;, Arizona, U.S.A.
}

\begin{abstract}
UBV magnitude differences in visual binaries are presented and their accuracy is discussed. Typical observed image profiles of double stars, including sample profiles of Antares, are shown.

This contribution will appear jointly with the earlier one (p. 20) in Lowell Obs. Bull., No. 154. The work has been supported by NSF Grant GP-6983.
\end{abstract}

\section{Discussion}

Heintz replies to questions by Laques and Nather on the Antares system. The current separation is $2^{\prime \prime} .8$ or $2^{\prime \prime} .7$; the motion is too slow to permit computation of an orbit, and the separation derived from an occultation observation by scanning in 1954 is considerably off from the photographic and visual positions.

Franz: Antares is a fairly wide pair, but the magnitude difference in the visual range is about 5 mag. At the altitude of Antares as observed from Flagstaff, the atmospheric dispersion is considerable. Fortunately the position angle is near $270^{\circ}$, otherwise the dispersion would have been troublesome.

Luyten: When the automatic Blink Survey starts we expect to have laser beam scans of our star images averaging probably 12 or 16 scans per star image. Hence we should get fairly accurate information on the diameters and areas of the stellar images. Normally, all this information would not be retained but a special effort might be worth while to retain it for double stars. We expect to find some tens of thousands of them. The main difficulty is going to be the fact that our plates are mainly $E$ plates taken through a red plexiglass filter. There are no known magnitude sequences for this combination.

Franz: This is probably an important source of information which has not yet been investigated. It would be a pity not to save data of this value though it might be expensive to retain them. The calibration should not be difficult to obtain.

Strand: A multiple exposure plate of Antares was taken at Johannesburg in the 'twenties'. It was measured in Leiden by practically every astronomer visiting Leiden in those years. On the basis of these measurements, Hertzsprung later evaluated the personal error of measurements in this kind of work.

Laques: Je voudrais savoir si vous apportez une correction aux mesures d'indice de couleurs tirées de vos enregistrements, corrections portant sur le fait de l'addition des flux lumineux qui se produit entre les deux composantes du couple et qui peut introduire une erreur de mesure?

Franz: I have assumed gaussian distribution and have added gaussians to represent the observed profile. I have not applied any corrections.

$S c a r f e$ : The combination of atmospheric dispersion with a large colour difference between the components could give an error in the observed separation. This would cause the amount by which your profiles overlap to be in error, and this results in an error of the $\Delta m$. Have you evaluated this error?

Franz: I am sure that the internal accuracy of the scanning technique is such that atmospheric dispersion could produce secondary effects that one might have to correct for by appropriate reduction techniques. Thus far I have not found such effects.

Franz replies to Fracastoro that interference filters could be used; the sensitivity of the scanner is high enough.

Strand remarks that the close agreement of $\pm 0.01 \mathrm{mag}$. with Eggen's measurements of $\Delta \mathrm{m}$ (as shown in Franz' paper) might be fortuitous, and based on too small a sample. Other comparisons show much larger errors of the Eggen results, for instance, an external mean error of $+\mathbf{0}^{m} .08$ is found from comparisons with Strand's measurements. Franz admits this possibility since only 9 pairs were used, and systematic effects were taken out. Batten suggests to observe some stars for which the $A m$ has been determined by Petrie's spectrophotometric method; it would be a valuable check on the latter method. 